\title{
HOY, CATALUÑA Y LOS JESUITAS. A PROPÓSITO DE UN LIBRO DE J. Mª BENÍTEZ I RIERA, S.I.
}

POR

Fernando de Lasala, S.I.

Pont. Università Gregoriana, Roma.

JOSEP M BENITEZ I RIERA, S.I., Jesuites i Catalunya: fets i figures, pròleg de Joan Bada Elías, epíleg d'Albert Mannent, Publicacions de l'Abadia de Montserrat (Scripta et documenta, 52), Barcelona 1996, 244 p., 8 ils., 2.800 pts.

Un libro en catalán sobre los jesuitas no es cosa frecuente en nuestros días. Desde los tiempos de la postguerra, cuando el P. Ignacio Casanovas dedico varios libros a la figura del fundador de la la Compañía de Jesús y al estudio de sus Ejercicios Espirituales, y cuando el $P$. Jose Calveras llegaba a ser una eminencia en el estudio del modo ignaciano de orar, han tenido que transcurrir muchos años para que apareciese este volumen. Me pregunto cuáles han sido los motivos. No han faltado excelentes escritores jesuitas, como los PP. Cándido de Da\}mases, especialista en historia jesuítica, en particular sobre san Francisco de Borja; Clemente Pujol, distinguido conocedor del Derecho Canónico de las Iglesias Orientales; y Miguel Badlori, recientemente galardonado con el Premio Príncipe de Asturias. Pero faltaba un escrito que tocase, al mismo tiempo, el núcheo de Cataluna y la esencia de la Compañía de Jesús.

No es fácil escribir un libro como éste. No basta agrupar unas páginas de aquí y de allá, poniéndolas en fila para que el lector las vaya recorriendo. Lo que ha ocurrido en este escrito es fruto de una sedimentada sintesis. Por un lado, el sentido de lo catalán se ha consolidado suficienternente como para poder entender y acoger este libro sin prejuicios. Por otro, la Compañía de Jesús, que se halla en circunstancias de urgente acomodación a los tiempos, y que mantiene enhiesto su pabellón a pesar del número menor de sus miembros, a través del P. Josep $M^{n}$ Benitez se presenta con sincera dignidad ante los catalanes.

No en vano es un hecho histórico que momentos transcendentales de la vida espiritual de Ignacio de Loyola fueron vividos en tierra catalana: si en Montserrat vel6 las amas, en Manresa se puso como un nif́o ante Dios como pedagogo y recibió el premio de la visión junto al Cardoner, y en Barcelona aprendio lecciones de latín con el maestro Ardevol, mientras aprendía a mendigar en las gradas de Santa María del Mar. El caballero vasco, educado en Arévalo, se consolido en Catalutha, antes de partir para Tierra Santa, para los estudios universitarios en Akcalá, Salamanca y Paris y para gobemar, finaimente, la Compañía en Roma. 
El P. Josep $M^{A}$ Benítez, profesor de Historia Modema en la Facultad de Historia Eclesiástica de la Pontificia Universidad Gregoriana de Roma, se ha decidido, ya era hora, a publicar cuatro conferencias y seis artículos, que complementa con cuatro apéndices, todos en tomo a la Compañía de Jesús en relación con Cataluña. Sus pretensiones no son, por el momento, hacer una historia exhaustiva de la presencia de esos religiosos en tierra catalana, sino presentar unos momentos de dicha historia, así como algunos de sus personajes: «fets i figures», es el subtítulo.

En primer lugar, aborda la historia de dos figuras que tuvieron mucho que ver con $\mathrm{Ca}$ taluña: Ignacio de Loyola y Francisco de Borja, virrey de Cataluña antes de ser jesuita y, después, tercer Superior General de la Compañía. «Manresa en la vida de San Ignacio», título del primer trabajo, es una conferencia que el P. Benítez pronunció en Manresa, con ocasión del cuarto centenario del nacimiento de San Ignacio. Aquellos once meses que el vasco convertido pasó en Manresa dejaron una huella imborrable, no sólo en su persona, sino en todos los que siguen el modo "ignaciano" de entender el Evangelio.

El Duque de Gandía y Virrey de Cataluña, Francisco de Borja, biznieto de Alejandro VI y del rey Fernando II de el Católico, es un personaje que se tiene de pie sin apoyatura alguna. Su esquinosa historia, vivida hondamente en el ambiente cortesano como caballero, esposo y padre; su concepción sobre la existencia y sobre la justicia humana; su decisión de ser jesuita tomada en Gandía después de hacer los Ejercicios Espirituales, tal como demuestra el $P$. Benítez, son retazos de su biografia trazados con rigor científico; pero a ellos se junta lo que el propio Autor escribe, y que me permito transcribir en traducción al castellano: «Una lectura ponderada de ta documentación sobre el período de virrey de Borja nos permite tener una visión global de lo que fue su acción de gobierno. Borja no aparece como un político original. Nunca llego a formular ningún proyecto de altura sobre Cataluña. Fue un ejecutor fiel, tenaz y honrado de las directrices dictadas por Carlos V. Se mantuvo en la línea del servicio imperial, identificada con el ideal hispánico», el cual era representado por Castilla, eje de la monarquía española.

El P. Benítez pasa luego a un caso particular, que no por ser más ceñido a un espacio menor deja de tener significado historico. Se trata de la dimensión pastoral de un profesor de filosofia y teologia de la Universidad de Cervera, el P. Pedro Ferrussola: solícito por los estudiantes pobres, por los gitanos perseguidos por Carlos III, por los moribundos, empeñado en la promoción del culto a la Virgen María bajo la advocación de la Inmaculada Concepción, prerrogativa mariana que los teólogos estaban estudiando por aquel entonces; devoto también de Nuestra Señor del Incendio, curiosa advocación local de María. El P. Ferrussola, promotor de la veneración al "Santo Misterio de Cervera", en relación con una reliquia del Lignum Crucis que allí se conserva y que fue traída por un soldado de los que participaron en el Saqueo de Roma de 1527 por orden de Carlos V. Todo lo que relata el $P$. Benitez está aseverado por las fuentes.

El tema de la expulsión de los jesuitas ha atraído siempre la atención de los enuditos, y de los no cultivados. En este punto merece que nos detengamos para agradecerle al $P$. Benítez su paciencia en adquirir, durante muchos años, tanta información que, casi sin querer, al pronunciar durante poco más de una hora una conferencia en el salón de la casa de Nuestra Señora di Monserrato, en Roma, dejo a los oyentes con la sensación de haber escuchado algo definitivo. Por eso, el profesor universitario, en este punto del libro, se hace todavía más fidedigno y verídico, porque se detiene, como quien sabe lo que dice, sobre las causas políticas, sociales, religiosas y de mentalidad que incidieron en la cruel medida tomada por Carlos III contra los

La Jglesia en la Encrucijada

Hispania Sacra 49 (1997) 
jesuitas. Vuelve a Cervera, y aborda el asunto de la expulsión concreta de los jesuitas allí residentes, y publica el informe oficial, hasta ahora inédito. Los jesuitas expulsados de España fueron embarcados en Salou y, habiendo sido rechazado su desembarque en Civitavecchia por Clemente XIII, quien no quería obedecer la orden unilateral del absolutista monarca español, fueron a parar a Bastia, Calvi y, en fin, a Bonifacio. El P. Benítez se ha servido de una crónica manuscrita, inédita, del último superior provincial de la Provincia jesuítica de Aragón, el zaragozano P. Blas Larraz. Finaliza este apartado sobre los acontecimientos del s. XVIII con un índice preparado por el catedrático y Canciller de la Universidad de Cervera, Don Ramón Lázaro de Dou y de Bassols, amigo de los jesuitas, a una obra inédita del P. Francisco de Isla que rebatía una carta pastoral del obispo de Burgos, Mons. José-Javier Rodríguez Arellano, en donde se alababa la decisión de Carlos III y se justificaba la necesidad de abolir absolutamente la "doctrina jesuita". Nos hallamos, pues, ante la cima del absolutismo monárquico del despotismo ilustrado, campo sobre el cual el profesor Benítez es uno de los mejores expertos.

Hasta aquí, los temas correspondian a la "antigua" Compañía de Jesús, es decir, la anterior a la supresión de 1773. A continuación nos presenta el P. Benítez, dando un salto cronológico, «El establecimiento de los jesuitas en Barcelona en la época de la Restauración», es decir, en 1881, con la fundación de su Colegio del Sagrado Corazón de Jesús en la céntrica calle de Caspe. Era el momento de la restauración monárquica en España. Este estudio resulta un modelo de concisión y de exactitud histórica. Y, luego, no podía faltar por parte de quien ha sido colaborador y amigo, un nuevo homenaje al P. Miguel Batllori, jesuita apreciado y discutido. Sobre él escribia el P. Ceferino Peralta, recientemente fallecido en Zaragoza y especialista en Baltasar Gracián, que "pasó su Patmos en Mallorca", recordando sus tiempos de la postguerra. Pues bien, el P. Benítez, a partir de sus primeros contactos con el P. Batllori, con respetuosa y delicada perspicacia, va trazando los rasgos fundamentales de su apretada biografia. No cae en adulaciones, a pesar de manifestarse muy afecto al biografiado. Sigue siendo crítico y objetivo. Redondea su exposición con dos "complementos", publicados con ocasión de los inicios de la «Obra completa» del viejo profesor, que esperar sacar en 19 volúmenes, y con motivo de concesión del Premio Príncipe de Asturias de Ciencias Sociales al que ya nos hemos referido arriba.

Un tema de conexión de los jesuitas catalanes con Roma es el estudio de la presencia catalana en la Pontificia Universidad Gregoriana; figuras destacadas desde el lejano siglo XVIII hasta hoy.

Finalmente, en el último capítulo, el Autor se centra en los profesores catalanes que han impartido lecciones en la mencionada Universidad Gregoriana. Al abrir ese tema, el P. Benítez trata de los primeros fundadores de dicha Universidad (Loyola y Borja). Presenta después al té́logo histórico-crítico P. Juan Bautista Gener, quien publicó su obra en Roma (1765-1775), y continúa con el filósofo y té́logo P. Joaquín Forn y Roget, quien, emigrado a Inglaterra durante los tiempos de la República Romana, regresó a Roma, en donde fue teólogo del obispo de la Seo de Urgel, el doctor José Cajxal, durante el Concilio Vaticano I; sigue el estudio con el conocido moralista de fin del siglo XIX, P. Valentín Casajoana; $y$, ya en el siglo XIX, presenta breves rasgos biográficos del moralista $P$. Pedro Vidal, sucesor del P. Francisco-Javier Wernz, famoso por el manual denominado comúnmente "WernzVidal"; sigue con el historiador P. José $M^{*}$ March, y con el canonista P. Miguel de los Santos Fábregas. Concluye dando las características esenciales de los cuatro profesores eméritos aún vivientes, sobre quienes, por serio, no sería oportuno tratar en plan histórico 
(PP. Clemente Pujol, Miguel Batllori, Antonio Queralt y José O'Callaghan), así como de otros cinco profesores todavf́a en activo en la Ciudad Etema.

A los diez capítulos se añaden apéndices que aumentan el grado de unidad del libro: una Cronología de historia de la Compañía de Jesús en Cataluña (del siglo XV al XX); una relación de los Padres Generales; la lista de todos los 105 superiores provinciales de las diversas configuraciones que ha tenido la Compañía de Jesús en el territorio de la antigua Corona de Aragón y del Principado de Cataluña; un directorio de todas las residencias, colegios o casas que los jesuitas han mantenido abiertas a lo largo de esos cuatro siglos y medio. El último apéndice es un "Nomenclator de los jesuitas catalanes más significativos", que da la biografia esencial de 154 de ellos, los más destacados por su acción pastoral, sea de orden intelectual, científica o académica, sea por su obra apostólica o social, sea por su fama de santidad, desde los primeros que todavía trataron al Fundador hasta los recientemente fallecidos. Es obvio que en el Nomenclator no se incluya a ningún jesuita vivo. A través de esta sabrosa lista, se puede percibir la universalidad de tales personas, quienes ejercieron el apostolado en todo el mundo: América hispana, Filipinas e India. Resulta un elenco muy completo: una especie de minibiografias que abarcan 44 páginas. Esto no había sido realizado hasta hoy con las figuras jesuíticas más importantes de Cataluña desde el siglo XVI, y merece todo agradecimiento. Por lo que respecta a los jesuitas de nuestro caduco siglo XX, el Nomenclator completa los datos biográficos del repertorio Escriptors jesuîtes de Catalunya. Bibliografia. 1931-1976 (editado por los PP. Antoni Borràs i Feliu, Francesc de Paula Sola i Carró, Josep M $^{\nwarrow}$ Torelló i Barenys), 1979.

El P. Benítez ha querido embellecer el volumen con adecuadas ilustraciones de las que merece la pena destacar, por su originalidad, la reproducción del bleo sobre tabla de Montserrat Gudiol, retrato de San Ignacio, ofrecido por la artista a la Compañía de Jesús en Cataluña, con ocasión del quinto centenario del nacimiento de Ignacio de Loyola y los 450 años de la fundación de la Orden. Pintura figurativa, cargada de psicología, que nos muestra un Ignacio peregrino, con ribetes casi franciscanos (Loyola era un admirador de Francisco) en sus meses de penitencia en Manresa. Otras ilustraciones incluyen los frontispicios de las obras Vita del P. Francesco Borgia (Roma 1616) del P. Pedro de Ribadeneira, y Theologia dogmatico-scholastica perpetuis prolusionibus polemicis historico-criticis sacrae antiquitatis monumentis illustrata (Roma 1767-1775), del P. Juan Bautista Gener; algunos retratos de jesuitas insignes y el mapa topográfico de los territorios comprados por los jesuitas en Barcelona para edificar el colegio, la iglesia y la residencia del Sagrado Corazón de Jesús, según la alineación exigida por los planes urbanísticos del segundo "ensanche" barcelones de finales del siglo XIX.

Joan Bada i Elias, catedrático de Historia Moderna en la Universidad de Barcelona, prologuista del libro, nos informa sobre la dedicación del P. Benítez a la Historia, en concreto a la historia de los jesuitas, compartiendo con él sus tareas docentes. El Epílogo de Albert Manent, demuestra la continuidad de los estudiosos de Historia dentro de la Compañía de Jesús, al mismo tiempo que un gran aprecio por ellos, aventurándose a decir que "Josep María Benítez forma parte de los jesuitas historiadores de nuestro siglo que han dejado o dejarán huella". Por nuestra parte, no nos queda decir sino que, a lo largo del libro, en cuya portada vemos la fachada de la familiar iglesia de Belén, en plenas Ramblas de Barcelona, Josep $\mathbf{M}^{a}$ Benítez ha demostrado, casi sin pretenderlo, que su ciencia consiste en saber lo que narra y en dar conclusiones certeras, cargadas de ese sentido común histórico que los catalanes denominan, sabiamente, el seny.

La Iglesia en la Encrucijada

Hispania Sacra 49 (1997) 